\section{Sternoclavicular joint}

Shoulder \& Elbow 2018, Vol. I0(4) 296-305 (C) The Author(s) 2018 Article reuse guidelines: sagepub.com/journals-permissions DOI: 10.1 I 77//7585732/8756880 journals.sagepub.com/home/sel

\author{
Rohit Dhawan', Rohit Amol Singh', Bernhard Tins' and \\ Stuart M. Hay ${ }^{1,2}$
}

\begin{abstract}
The sternoclavicular joint is a saddle shaped, synovial joint and is the only skeletal articulation between the axial skeleton and the upper limb. Here, a reviewis provided of the anatomy, biomechanics, traumatic and atraumatic conditions, and management options for the various conditions described.
\end{abstract}

\author{
Keywords \\ anatomy, instability, osteoarthritis, SAPHO, sternoclavicular joint, traumatic injuries
}

Date received: 26th September 2017; revised: 14th December 2017; accepted: 3Ist December 2017

\section{Anatomy of the Sternoclavicular Joint}

The sternoclavicular joint (SCJ) (Fig. 1) is a saddle shaped, synovial joint and is the only skeletal articulation between the axial skeleton and the upper limb. The medial end of the clavicle has a larger surface area and thicker hyaline cartilage than the adjacent sternum. The clavicle articulates with the reciprocal notch of the sternum and the superior surface of the first costal cartilage. The joint is concave in the vertical axis and convex in the anteroposterior axis. The articulating surfaces are not fully congruent and divided by an articular disc. The articular disc attaches to the posterosuperior aspect of the medial articulating surface of the clavicle and the anterosuperior aspect of the first costal cartilage. The rest of the disc is covered by capsule. The disc is thicker in the periphery and at the attachment sites. As a result of the sella turcica-like shape of the joint, the disc has mobility in the anteroposterior and vertical axis. The movement involving elevation and depression occurs between the articular disk and clavicle, whereas protraction and retraction occurs between articular disc and the sternum. ${ }^{1}$

The SCJ is one of five articulations of sorts that influence the pattern of movement of the shoulder girdle. Although diminutive in size, symptomatic pathology will impact upon scapulothoracic and glenohumeral rhythm; the treatment of which may successfully restore normality. The normal SCJ achieves $35^{\circ}$ of movement both in the coronal and horizontal plane during shoulder abduction along with $45^{\circ}$ of rotation in its long axis. ${ }^{2}$ The ligaments around the SCJ include the anterior sternoclavicular ligament, posterior sternoclavicular ligament, the costoclavicular ligament and the interclavicular ligament.

\section{Biomechanics of the SCJ}

The anterior sternoclavicular ligament is broad and extends from the anterosuperior surface of the medial end of the clavicle to the upper anterior edge of the manubrium and first costal cartilage. The posterior sternoclavicular ligament extends from the posterior aspect of the sternal end of the clavicle to the posterior superior aspect of the manubrium. The posterior ligament confers primary stability to the joint, which is supplemented by the rest of the ligaments. The interclavicular ligament extends between the medial ends of the clavicle and is continuous with the deep cervical fascia superiorly. The costoclavicular ligament (Figs 2 and 3) is shaped like a short and flattened, inverted cone. It has two laminae present on the anterior and posterior aspects of the clavicle. The anterior lamina is lateral and attached to

\footnotetext{
'Robert Jones and Agnes Hunt Orthopaedic and District Hospital NHS Trust, Gobowen, Oswestry, UK

${ }^{2}$ Royal Shrewsbury Hospital, Shrewsbury, Shropshire, UK

Corresponding author:

Rohit Dhawan, Robert Jones and Agnes Hunt Orthopaedic and District Hospital NHS Trust, Gobowen, Oswestry, SYIO 7AG, UK.

Email: rohitkrdhawan@yahoo.com
} 


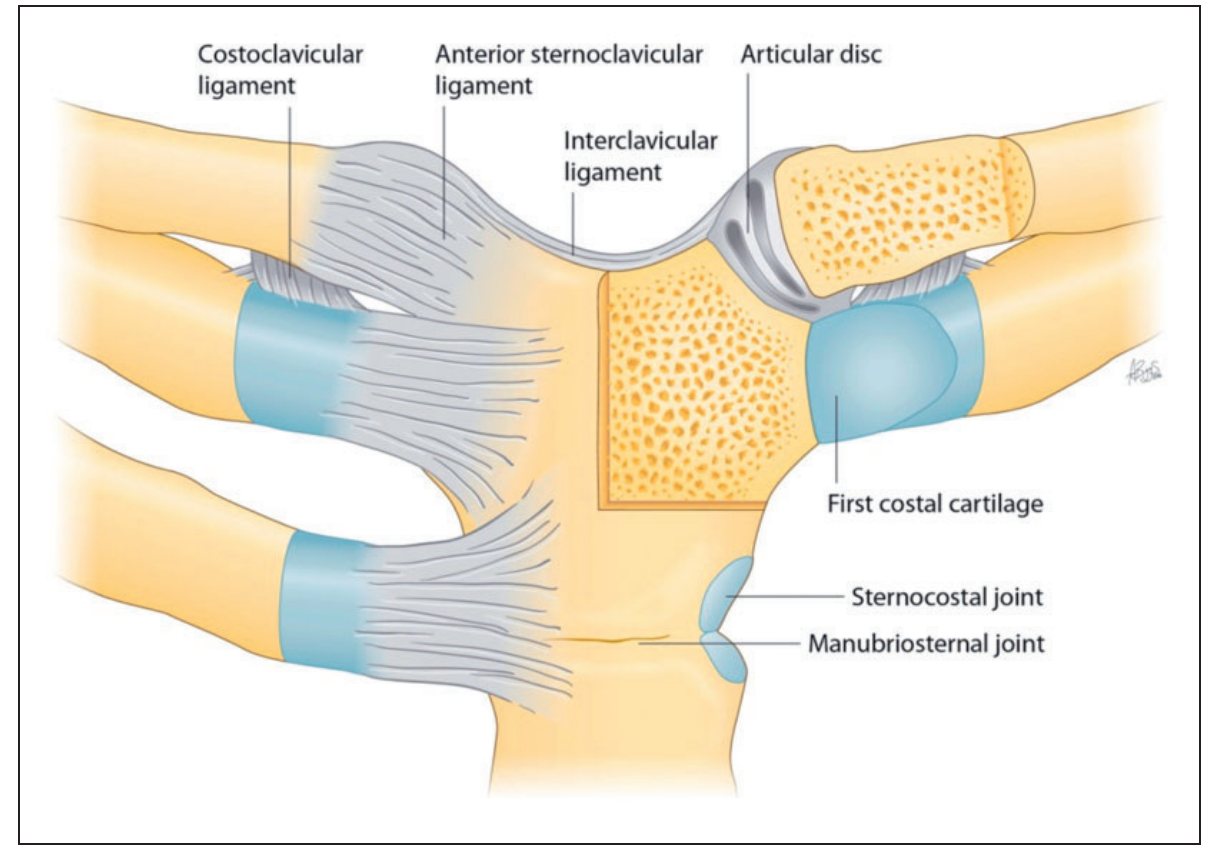

Figure I. Anatomy of the sternoclavicular joint.

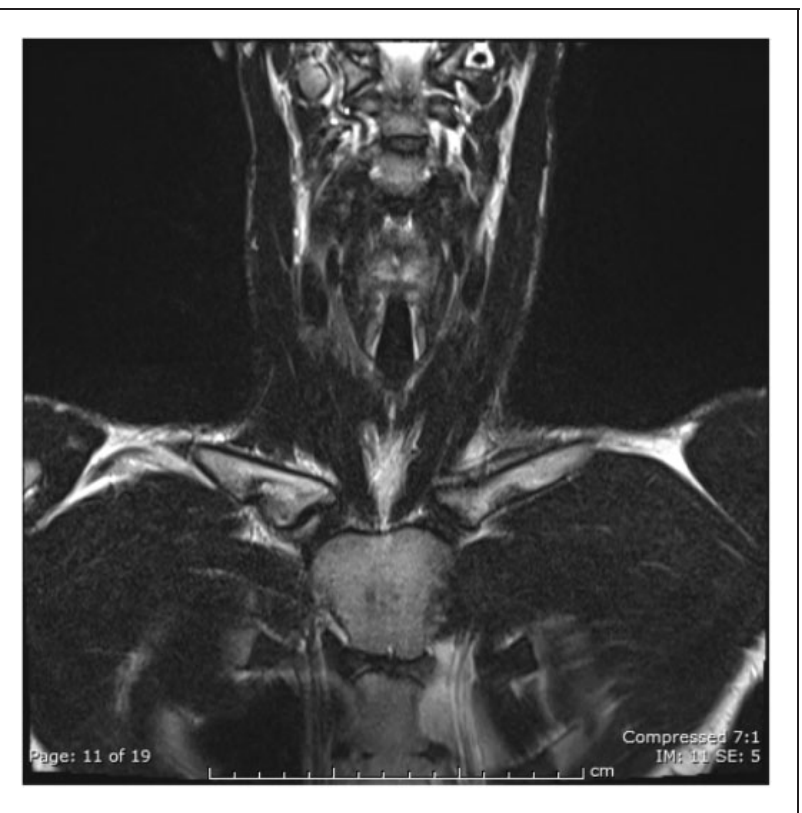

Figure 2. Bilateral very prominent insertion of costoclavicular ligament.

the upper aspect of first rib and costal cartilage inferiorly and the inferior aspect of medial clavicle superiorly. The posterior lamina is medial and attached to the first rib and costal cartilage in a similar fashion to the anterior lamina. Sometimes, the attachments of the ligaments can be very prominent (Fig. 3). This should not be

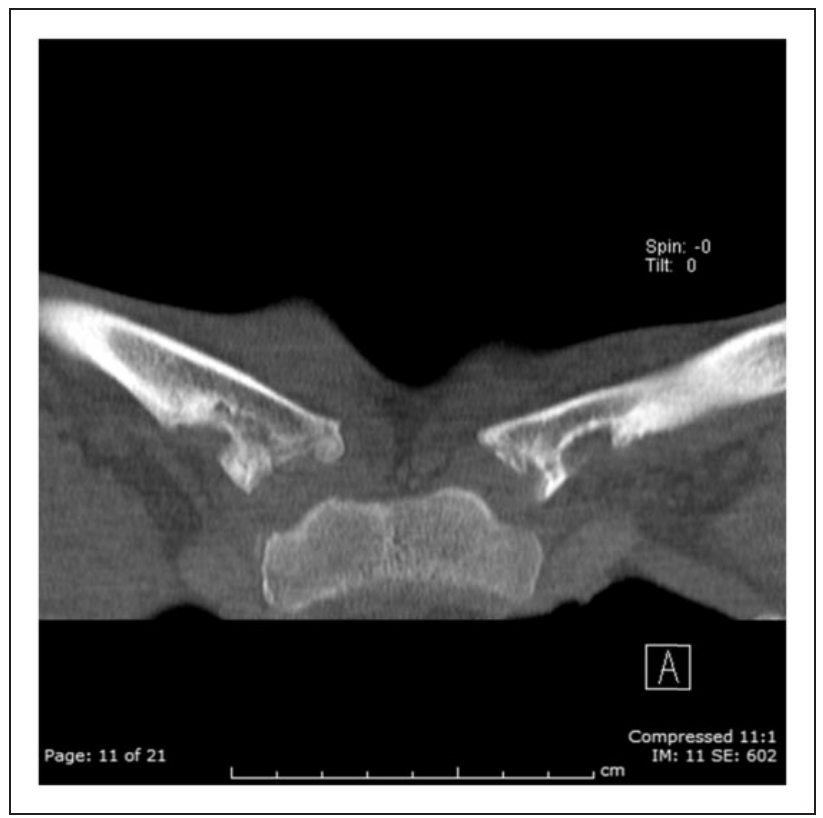

Figure 3. Bilateral very prominent insertions of costoclavicular ligaments - changes such as this are typical for a normal variant and should not be mistaken for an aggressive destructive process.

mistaken for pathology. Force is transmitted through the clavicle to the ligaments on the medial aspect and more often the clavicle will fracture before the surrounding ligaments fail.

Vascular supply to the SCJ comes from the suprascapular artery and internal thoracic artery. 
Nerve supply of the joint is from the medial suprascapular nerve and the nerve to the subclavius. The brachiocephalic trunk, common carotid artery and the internal jugular vein all lie directly posterior to the SCJ.

The joint is difficult to assess on routine chest radiographs. A computed tomography (CT) scan is a better option to get a three-dimensional understanding of the SCJ. On magnetic resonance imaging (MRI), the articulating surfaces and intra-articular disc are better seen in coronal sequences, whereas axial sequences depict the anterior and posterior capsule and ligaments. The sagittal sequences are useful in assessing the costoclavicular ligaments. ${ }^{3}$

\section{Traumatic injuries of the SCJ}

The injuries of the SCJ include both fractures of the medial end of clavicle and dislocation of the joint. The incidence of dislocation of the SCJ is uncommon compared to that of the glenohumeral or acromioclavicular joint. SCJ dislocations comprise of $1 \%$ of all dislocations and $3 \%$ of upper limb dislocations. ${ }^{4-6}$ Based on severity, SCJ injuries are classified into type 1 - sprain of SCJ without laxity or pain; type 2 - rupture of SCJ ligaments, costoclavicular ligaments stay intact; and type 3 - rupture of SCJ and costoclavicular ligaments resulting in dislocation of the joint. ${ }^{7}$ Furthermore, SCJ instability can be classified according to directions - anterior, posterior and superior; cause traumatic and atraumatic; and duration - acute and chronic.

\section{Mechanism of Injury}

Dislocation of the SCJ can also be described by the direction of the applied forces.

Anterior dislocation. If force is applied in posterolateral direction and the shoulder rolled backwards, the clavicle levers on the underlying first rib, resulting in anterior dislocation of the medial end of the clavicle. Anterior dislocations (Fig. 4) are more common than posterior dislocations. ${ }^{8}$ Indirect forces represent a more common mechanism leading to SCJ dislocation because direct forces on the medial end of clavicle account for only $10 \%$ to $25 \%$ of SCJ dislocations. ${ }^{9}$ The most common cause of dislocation of SCJ is road traffic accidents followed by sports-related injuries.

Posterior dislocation. Direct application of force on the medial clavicle pushes it posteriorly behind the sternum into the mediastinum. This can happen in a variety of ways, including a supine athlete being jumped upon and hit on the medial clavicle by the knee of another athlete, a patient being ran over by a vehicle, a direct

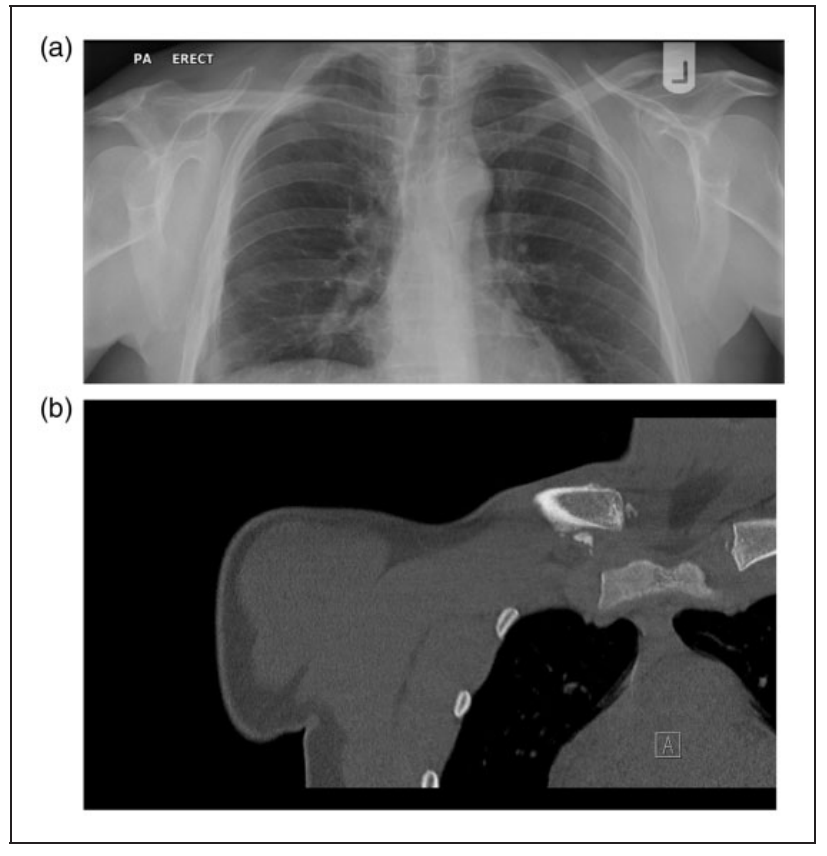

Figure 4. (a) Dislocation right sternoclavicular joint (SCJ). (b) Dislocation right SCJ.

kick to medial clavicle or a patient being crushed between a stationary object and a moving vehicle.

Indirect application of force to the SCJ can also result in anterior or posterior dislocation of the joint. If force is applied in an anterolateral direction and the shoulder rolled forward during lateral compression, the medial end of clavicle dislocates posteriorly.

Posterior dislocation of the SCJ are rare but can have devastating consequences including pneumothorax, dysphagia, hoarseness, vascular injury and injury to brachial plexus. ${ }^{10-17}$

\section{Clinical Presentation}

The patient normally presents with pain on the medial aspect of clavicle after sustaining a fall on the ipsilateral shoulder. Severe pain and reduced movement of the shoulder joint accompany SCJ dislocations. Lateral compression of the shoulders increases the severity of pain. The patient normally supports the injured arm with the other uninjured arm. The shoulder on the side of the fractured clavicle or dislocation appears shortened and thrust forward with the head of the patient tilted towards the dislocated clavicle. Clinical examination to ascertain the type of injury can be difficult as a result of excessive swelling that can hide the prominence of the medial clavicle or depression of the SCJ. The patient is better examined in a supine position; however, this could be difficult because laying the patient down can exacerbate symptoms. 
For posterior dislocations, as a result of the close proximity of the joint with the major vessels, mediastinum, brachial plexus, oesophagus and trachea, patients can exhibit a myriad of symptoms, including compromised circulation to the arm, brachial plexus symptoms, dysphagia, odynophagia, dyspnea, hoarseness and choking. The examining clinician should maintain a high index of suspicion to diagnose the injury and order appropriate investigations for diagnosis.

The medial epiphysis of the clavicle ossifies between 18 years and 20 years and fuses between 23 years and 25 years. This should be kept in mind when analyzing images of the joint. ${ }^{18}$ A meta-analysis by Tepolt et al. ${ }^{19}$ on an adolescent population revealed that $69 \%$ of patients with medial SCJ injuries had a physeal fracture and $23 \%$ of the injuries were true dislocations. This is because the medial epiphysis is the weakest structure in the region, resulting in failure. Closed reduction performed within $48 \mathrm{~h}$ of the injury had better outcomes than those performed after $48 \mathrm{~h}$. After closed reduction, the joint is splinted using a figure of eight bandage for 4 weeks.

Associated injuries can include fractures of the ribs and scapula and can be a variant of scapular-clavicularthoracic syndrome. ${ }^{20}$ Being a part of high-energy trauma, the diagnosis could be delayed as a result of distraction by lower limb or spinal injuries. Brachial plexus injuries can be temporary or permanent. Vascular injury involving brachiocephalic or subclavian vessels can range from asymptomatic compression to a vascular laceration by the fractured clavicle. Intimal injuries can lead to thrombosis with subsequent occlusion of the vessels. ${ }^{21}$ Injury to major vessels including internal mammary artery, superior vena cava and brachiocephalic vein has been reported to result in paediatric deaths. ${ }^{22,23}$

\section{Treatment of SCJ Dislocation and Instability}

Occasionally asymptomatic SCJ subluxation is diagnosed amongst individuals with joint laxity and often these can be managed - at least initially, conservatively with physiotherapy.

\section{Anterior dislocation: closed reduction}

Standard treatment for anterior dislocation is nonoperative management. For reduction of anterior dislocation, patient is placed supine on the operating table with a sandbag between the scapulae. Following this, posterior force is applied on the medial end of the clavicle along with abduction of shoulder to $90^{\circ}$ and extension of $10^{\circ}$ to $15^{\circ}$. A figure of eight bandage is applied thereafter to hold the joint in place for a few weeks. ${ }^{24-26}$

\section{Posterior dislocation: closed reduction}

Closed reduction for posterior dislocation involves abduction of shoulder and applying traction. At the same time, the ipsilateral shoulder is extended to assist in anterior translation of the medial end of clavicle. $^{25}$

\section{Open reduction and internal fixation}

For both anterior and posterior dislocation, open stabilization can be performed through a transverse incision over the SCJ and going through the platysma and elevating pectoralis major and sternocleidomastoid as necessary. Care must be taken to protect the supraclavicular nerves.

Various techniques have been described for stabilizing the joint. These include repair using suture anchors, reconstruction using autologous or synthetic grafts and internal fixation. Sutures can be drilled through clavicle and fastened in a loop. ${ }^{27}$ In this study, patients were followed up for a mean of 4.5 years and had fair outcomes. Franck et al. ${ }^{28}$ reported the usage of a Balser plate fixed to clavicle and manubrium sterni to treat SCJ dislocation. Several different types of grafts including synthetic grafts, gracilis tendon, semitendinosus and fascia lata have been used to form a figure of eight stitch between clavicle and the manubrium or first rib. ${ }^{29-31}$ Booth and Roper $^{32}$ have described looping the sternocleidomastoid through the first rib and sutured back to the costoclavicular ligament. All of the patients resumed sporting activities. The fixation technique of using k-wires across the SCJ has shown an approximate death rate of $40 \%$ as a result of vascular complications because of wire migration. ${ }^{33}$ Apart from Abiddin et al., ${ }^{27}$ the rest of the studies describe early follow-up results up to 2 years postoperatively. Burrows $^{34}$ described tenodesis of subclavius tendon for recurrent dislocation of the SCJ together with capsulorrhaphy. Semitendinosus or sternocleidomastoid muscle tendon can be used as a figure of eight ligament reconstruction across the SCJ. ${ }^{35-37}$ This has been shown to be biomechanically superior to intramedullary fixation. ${ }^{38}$

\section{Osteoarthritis}

Osteoarthritis (OA) (Fig. 5) of the SCJ is relatively common, although asymptomatic in most cases, postmortem studies in patients aged over 60 years have shown OA to be present in over $50 \%{ }^{39}$ A recent CT scan based study found the evidence of at least one feature of $\mathrm{OA}$ in all the patients above 61 years of age. The prevalence of SCJ OA was $89 \%$ in patients older than 50 years as opposed to $9 \%$ in patients less than 50 years. ${ }^{40}$ It is more common in postmenopausal 


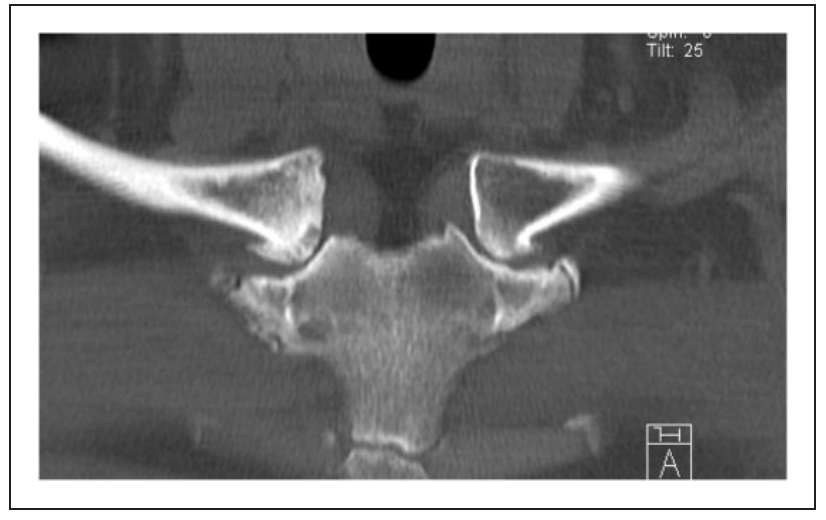

Figure 5. Osteoarthritis of bilateral sternoclavicular joint - loss of joint space, sclerosis, marginal osteophyte formation and gas in the joint space.

women and manual labourers. Pain with OA of the SCJ is typically provoked during abduction or forward flexion beyond $90^{\circ}$. The radiographic features typical of OA are: loss of joint space, subchondral cysts, subchondral sclerosis and marginal osteophytes, which are typically located over the inferior aspect of the medial clavicle. ${ }^{39}$ Osteopenia is not a typical feature.

Treatment is usually conservative with anti-inflammatories and/or injections of steroid. Surgery is recommended for symptomatic patients unresponsive to conservative measures. Surgical options of treatment include arthroscopic and open resection arthroplasty. ${ }^{41}$ Interpositioning of joint with the sternal head of sternocleidomastoid following excision of the medial SCJ has been described with satisfactory outcomes. ${ }^{42}$ Open resection arthroplasty of the medial end of clavicle with short-term follow-up has been reported to have a higher return to sport activities along with a revision rate of $17 \%{ }^{43}$

\section{Inflammatory Arthritides of the SCJ}

The SCJ is susceptible to a spectrum of inflammatory conditions that can present as acute or chronic episodes. In the assessment of an inflammatory arthritis it is important to ascertain a detailed social and family history including medications and recreational drugs. Clinically, there may be localized tenderness, bony enlargement and generic signs of inflammation.

A significant proportion of patients with rheumatoid arthritis (RA) are found to have intra-articular disc degeneration. ${ }^{3,44}$ The early stages have little in the way of radiological changes, with bony erosions and joint destruction occurring in chronic severe cases. Rheumatoid arthritis of the SCJ is more common in women and is often positive for rheumatoid factor/antinuclear antibodies. The treatment remains mainly medical with medial clavicle excision reserved for symptomatic cases resistant to medical treatment. ${ }^{45}$

A variety of seronegative spondyloarthropathies such as psoriatic arthritis, Reiter's syndrome and ankylosing spondylitis can involve the SCJ. ${ }^{46-48}$ The SCJ can be involved in $90 \%$ of severe cases of psoriatic arthropathy and can be a presenting symptom in $50 \%$ of cases compared to $4 \%$ of cases in ankylosing spondylitis. $^{49,50}$ Seronegative spondyloarthropathies are more common in younger men, with HLA B27 being a diagnostic feature.

In the evaluation of a swollen SCJ, the aspirate may be examined under polarizing light to show either positively or negatively birefringent crystals representing pseudogout and gout, respectively, which can both affect the SCJ. ${ }^{51}$

The treatment for all these conditions remains largely medical with first line anti-inflammatories and disease modifying drugs for seropositive arthritis and resistant cases of seronegative spondyloarthropathy and long term prophylaxis with xanthine-oxidase inhibitor for crystal deposition arthropathy. ${ }^{52}$ Untreated chronic inflammation of the SCJ leads to restricted mobility and secondary degenerative joint changes.

The general principles of investigation of inflammatory arthritides include conventional radiographs. Depending on the underlying pathology, the radiographic findings may vary. In RA, bone destruction with erosion, osteopenia and adjacent soft tissue swelling is seen. In crystal arthropathies, reactive osteopenia is not usually seen; instead, soft tissue calcification may be noted. ${ }^{53} \mathrm{CT}$ can be useful for evaluating ossifying lesions, whereas MRI gives better depiction of inflammatory soft-tissue enlargement and bone marrow change of the medial clavicle.

\section{Sclerosing Conditions of the SCJ and Surrounding Structures \\ SAPHO}

SAPHO syndrome (Fig. 6) is a group of heterogenous disorders that can be difficult to diagnose if there are no skeletal manifestations. SAPHO stands for Synovitis, Acne, palmar or plantar Pustulosis, Hyperostosis and Osteitis. The SCJ is the most common location of the disorder followed by the sacroiliac joint and spinal lesions. SAPHO has overlapping symptomatology compared to spondyloarthropathy. Up to $30 \%$ of SAPHO patients have a positive HLA B27. ${ }^{54}$

Radiologically, SAPHO typically presents as florid hyperostosis with an inflammatory component. Lesions in sacroiliac joints lead to erosion of the joint followed by sclerosis and hyperostosis. Ossifications in the 


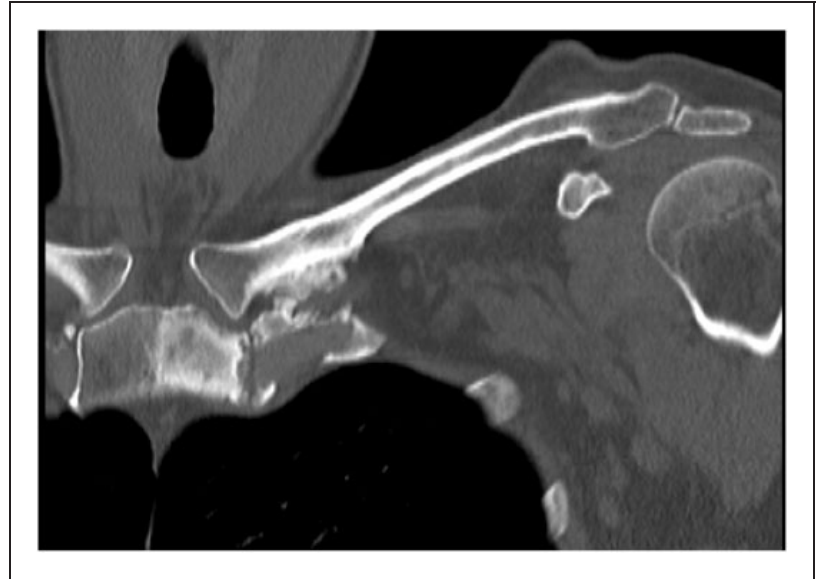

Figure 6. Coronal view of sternoclavicular joint showing hyperostosis.

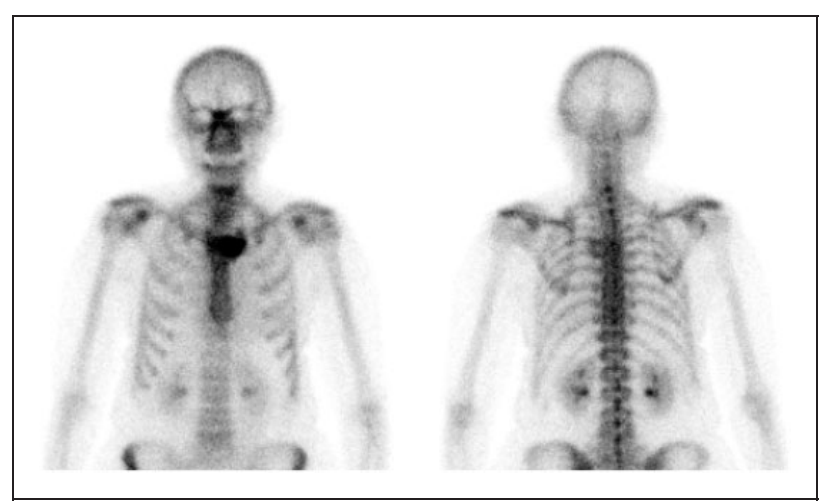

Figure 7. 'Bull's horn' appearance on bone scan.

paravertebral region can be mistaken for syndesmophytes. As a result, SAPHO can sometimes be mistaken for ankylosing spondylitis. ${ }^{55-58}$ However, SAPHO is usually multifocal rather than generalized and also affects the diaphysis of long bones, which is atypical for ankylosing spondylitis. Similarly, ankylosing spondylitis almost always affects the sacroiliac joints first before other areas are affected. When affecting the SCJ, the classic picture of SAPHO looks like a 'bull's horn' (Fig. 7) on nuclear medicine studies because of increased uptake of radioactive tracer at the SCJ bilaterally. SAPHO also often affects the mid clavicle; the differential diagnosis includes infection and malignancy. MRI images show oedema-like changes in the bone indicating inflammation, whereas CT demonstrates hyperostosis and bone scans show increased activity; these are the findings typical for osteitis.

Treatment of SAPHO usually involves nonsteroidal anti-inflammatory drugs, corticosteroids, bisphosphonates and sulphasalazine. Refractory SAPHO has been treated with anti-tumour necrosis factor medications including infliximab. ${ }^{59-61}$

\section{Condensing osteitis}

Condensing osteitis is a rare condition described by Brower in 1974. As described by Brower ${ }^{62}$, there is pain 'along the top of the shoulder' that becomes aggravated by abduction of the arm. Usually, patients present with a painful SCJ and fusiform tender swelling over the joint. Radiologically, there is sclerosis seen on the medial end of clavicle, usually on the inferior margin. Articulating surfaces are normal and there is no evidence of bone destruction on radiographic imaging. Radionuclide bone scan reveals localized tracer uptake. Histology reveals increased normal bone formation and reinforcement of cancellous bone along with obliteration of marrow spaces. Some consider the title to be a misnomer because there is no inflammation seen in the condition. An alternative term suggested is 'post-traumatic clavicular sclerosis'. ${ }^{62}$ These changes are presumed to be a result of abnormal or increased stresses through the SCJ. The differential diagnoses include osteoid osteoma, sternocostoclavicular hyperostosis $(\mathrm{SCCH})$, septic arthritis of SCJ and avascular necrosis of the medial end of clavicle. In SAPHO, there is often soft tissue inflammation that actually helps to suggest its diagnosis. However condensing osteitis and SCCH may be variants of SAPHO, in particular representing a later, quiescent phase.

\section{Friedrich's disease}

Condensing osteitis can be confused with avascular necrosis of the medial end of clavicle, also known as Friedrich's disease. This usually affects adolescent and paediatric patients. Clinically, the presenting symptoms could be similar to condensing osteitis. This has been debated by some authorities who consider this to be no different than condensing osteitis. ${ }^{63-65}$ However, clinical symptoms in patients with Friedrich's disease can be relatively shorter compared to condensing osteitis. Radiographic findings show involvement of the entire medial end of the clavicle with subchondral irregularities and defects in the articulating surfaces. The hallmark of Friedrich's disease is presence of necrotic bone, fibrosis of marrow spaces and haversian canal along with the presence of empty lacunae. ${ }^{62}$ Management involves the provision of nonsteroidal anti-inflammatory medications. Excision of the medial end of clavicle is the last choice if conservative management fails.

\section{Infection/tumour}

A swelling (painful or painless) on the medial aspect of clavicle can be worrisome. It would be prudent to 
exclude either infection or tumour in the SCJ despite $\mathrm{OA}$ and the other inflammatory arthritides being the most common diagnoses. Clinically, the differentiating factors could be a short history of presentation, increase in size, extremes of age, presence of other primary tumours and involvement of nondominant side. The presence of primary or metastatic cancer in the SCJ has been reported in the literature. ${ }^{66,67}$

Infection of the SCJ is a rare entity that is monoarticular and insidious in onset. Clinical presentation is that of low grade fever, shoulder girdle discomfort, erythema, warmth and swelling of the SCJ. This can lead to abscess formation in one-fifth of patients and concomitant sternal osteomyelitis in half of the patients, with the usual organisms being Staphylococcus aureus and Pseudomonas aeruginosa. ${ }^{68}$ Complications of septic arthritis of the joint include septic shock, superior vena cava syndrome and mediastinitis. Infection is seen more often in intravenous drug abusers, diabetics and patients with rheumatoid arthritis. When arriving at this diagnosis, it is important to distinguish it from SAPHO syndrome and tumour, which might include osteosarcoma, Ewing's sarcoma, lymphoma and metastatic disease.

In infection, MRI is the investigation of choice to assess the joint and neighbouring structures. MRI findings include joint space widening, joint destruction and sometimes adjacent gas or fluid collection. CT may be used as an adjunct to MRI and depicts bone destruction better.

Methods of treatment include antibiotics, as well as surgical debridement with or without negative pressure therapy, allowing the wound to heal by secondary intention. Alternatively, treatment comprises simultaneous debridement, resection of the medial end of clavicle and muscle flap coverage using pectoralis major, latissimus dorsi or rectus abdominis muscle. ${ }^{69-73}$. Resection of the medial end of clavicle followed by suturing of the residual clavicle to the periosteum of the first rib is another option. During excision of the medial end of the clavicle, the remnants of intra-articular ligaments and capsule are preserved.

Recently, newer techniques have been described, which include initial debridement and then delayed bone resection and pectoralis major flap reconstruction. ${ }^{74}$ The differential diagnosis of SAPHO, infection and tumour is not always easy, although, clearly, this has important management and prognostic implications and needs to be established by investigation, including biopsy if necessary.

\section{Imaging Considerations}

Usually, radiographs are the first imaging investigation of choice in orthopaedics and, usually, images are acquired in two orthogonal planes.
The SCJ is a complex joint on the anterior chest wall. Orthogonal views are difficult here and the routine examination consists of anteroposterior and anteroposterior oblique views. This does lead to partial superimposition of bone structures. In particular, the oblique views rely on the skill of the radiographer. Nevertheless, major malalignment, destruction, bone trauma or OA should be visible.

To assess the SCJ, the serendipity view has usually been used. This is a radiographic projection centred on the SCJ with $40^{\circ}$ cranial angulation. This view should include the medial third of both clavicles. Normally, the medial ends of the clavicles are equidistant from the sternum and in the same horizontal plane. In posterior dislocation, the dislocated clavicle will be projected caudal to the mean horizontal plane of the SCJ; in anterior dislocation, it will be projected cranially.

Nowadays, this is often superseded by CT if there is serious concern regarding SCJ malalignment.

As in other parts of the body, a more sensitive imaging investigation for bone and soft tissue change is MRI. Any inflammation will show up as increased fluid signal, be it in the bone marrow or the soft tissues. MRI can assess the joint for alignment and can assess the integrity of the capsular and ligamentous structures. Bone marrow infiltration is reliably seen only on MRI but not with CT. Whole body MRI may be indicated in the assessment of certain lesions such as multiple myeloma or chronic recurrent multifocal osteomyelitis (CRMO) or SAPHO.

$\mathrm{CT}$ is the best modality for the assessment of bone integrity and alignment. In the trauma setting, CT of the chest would automatically include the SCJ and allow for its assessment. CT can also assess bone destruction or new bone formation in tumors but, generally, MR is preferable for tumor imaging because it enables the assessment of marrow infiltration and soft tissue extension more reliably than CT.

Nuclear medicine techniques mainly comprise bone scintigraphy with Technetium ${ }^{99} \mathrm{~m}$-MDP and positron emission tomography (PET) imaging. Standard planar bone scintigraphy with Technetium ${ }^{99} \mathrm{~m}$-MDP images phosphate metabolism and therefore bone turnover. This is often combined with three-dimensional acquisition of the scintigraphy phase and projected on to a conventional CT scan.

PET imaging is usually performed with fludeoxyglucose, a glucose analogue and images glucose metabolism rather than bone metabolism, although there are now other PET tracers available to image bone metabolism, especially F18-fluoride.

\section{Pet Imaging is also Usually Combined with a CT Scan}

Nuclear medicine techniques are particularly useful if the wider skeleton is to be investigated for lesions for 
example to determine whether a bone lesion is single or not. Therefore, suitable applications include the assessment of metastatic disease, inflammatory arthropathies and spondylarthropathies including SAPHO, CRMO and possibly of infection. In SAPHO, the typical 'bull's horns' configuration may be visible. In CRMO, MRI is generally preferred because of the 'radiation dose' of nuclear medicine techniques.

Ultrasound is generally not the first investigation of choice. However, in our practice, ultrasound is sometimes performed for a 'lump' on the anterior superior chest wall and then typically demonstrates local osteophyte formation in the OA of the SCJs. Occasionally, inflammatory arthritis or very rarely infection may be seen sonographically. Alignment can also be assessed with ultrasound, although ultrasound is operator dependent and ultrasound images are not as easily interpreted as any of the other imaging modalities.

\section{Declaration of Conflicting Interests}

The author(s) declared no potential conflicts of interest with respect to the research, authorship, and/or publication of this article.

\section{Funding}

The author(s) received no financial support for the research, authorship, and/or publication of this article.

\section{Ethical Review and Patient Consent}

Not required for this article.

\section{References}

1. Inman VT, Saunders JB and Abbott LC. Observations of the function of the shoulder joint. Clin Orthop Relat Res 1944; 330: 3-12.

2. Philipson MR and Wallwork N. (iii) Traumatic dislocation of the sternoclavicular joint. Orthop Trauma 26: 380-384.

3. Higginbotham TO and Kuhn JE. Atraumatic disorders of the sternoclavicular joint. J Am Acad Orthop Surg 2005; 13: $138-145$.

4. Renfree KJ and Wright TW. Anatomy and biomechanics of the acromioclavicular and sternoclavicular joints. Clin Sports Med 2003; 22: 219-237.

5. Kocher MS, Dupré MM and Feagin JA. Shoulder injuries from alpine skiing and snowboarding. Aetiology, treatment and prevention. Sports Med 1998; 25: 201-211.

6. Kocher MS and Feagin JA. Shoulder injuries during alpine skiing. Am J Sports Med 1996; 24: 665-669.

7. Allman FL Jr. Fractures and ligamentous injuries of the clavicle and its articulation. J Bone Joint Surg Am 1967; 49: 774-784.

8. Nettles JL and Linscheid RL. Sternoclavicular dislocations. J Trauma 1968; 8: 158-164.

9. Mehta J, Sachdev A and Collins J. Retrosternal dislocation of the clavicle. Injury 1973; 5: 79-83.
10. Worman LW and Leagus C. Intrathoracic injury following retrosternal dislocation of the clavicle. J Trauma 1967; 7: 416-423.

11. Jain S, Monbaliu D and Thompson JF. Thoracic outlet syndrome caused by chronic retrosternal dislocation of the clavicle. Successful treatment by transaxillary resection of the first rib. J Bone Joint Surg Br 2002; 84: 116-118.

12. Howard FM and Shafer SJ. Injuries to the clavicle with neurovascular complications. A study of fourteen cases. J Bone Joint Surg Am 1965; 47: 1335-1346.

13. Nakayama E, Tanaka $T$, Noguchi $T$, Yasuda $J$ and Terada Y. Tracheal stenosis caused by retrosternal dislocation of the right clavicle. Ann Thorac Surg 2007; 83: 685-687.

14. Gardner MA and Bidstrup BP. Intrathoracic great vessel injury resulting from blunt chest trauma associated with posterior dislocation of the sternoclavicular joint. Aust NZ J Surg 1983; 53: 427-430.

15. Mirza AH, Alam K and Ali A. Posterior sternoclavicular dislocation in a rugby player as a cause of silent vascular compromise: a case report. Br J Sports Med 2005; 39: e28.

16. Borowiecki B, Charow A, Cook W, Rozycki D and Thaler S. An unusual football injury. Arch Otolaryngol 1972; 95: 185-187.

17. Camara EHS, Bousso A, Tall M and Sy M. Posterior sternoclavicular dislocations. Eur J Orthop Surg 2009; 19: 7. https://doi.org/10.1007/s00590-008-0363-0.

18. Schulz R, Mühler M, Mutze S, Schmidt S, Reisinger W and Schmeling A. Studies on the time frame for ossification of the medial epiphysis of the clavicle as revealed by CT scans. Int J Legal Med 2005; 119: 142-145.

19. Tepolt F, Carry PM, Taylor M and Hadley-Miller N. Posterior sternoclavicular joint injuries in skeletally immature patients. Orthopaedics 2014; 37: e174-e181.

20. Laffosse J-M, Espié A, Bonnevialle N, et al. Posterior dislocation of the sternoclavicular joint and epiphyseal disruption of the medial clavicle with posterior displacement in sports participants. J Bone Joint Surg Br 2010; 92: 103-109.

21. Benitez CL, Mintz DN and Potter HG. MR imaging of the sternoclavicular joint following trauma. Clin Imaging 2004; 28: 59-63.

22. Fenig M, Lowman R, Thompson BP and Shayne PH. Fatal posterior sternoclavicular joint dislocation due to occult trauma. Am J Emerg Med 2010; 28: 385.

23. Cooper GJ, Stubbs D, Waller DA, Wilkinson GA and Saleh M. Posterior sternoclavicular dislocation: a novel method of external fixation. Injury 1992; 23: 565-566.

24. Kirby JC, Edwards $\mathrm{E}$ and Kamali Moaveni A. Management and functional outcomes following sternoclavicular joint dislocation. Injury 2015; 46: 1906-1913.

25. Rockwood CA, Groh GI, Wirth MA and Grassi FA. Resection arthroplasty of the sternoclavicular joint. J Bone Joint Surg Am 1997; 79: 387-393.

26. Sewell MD, Al-Hadithy N, Le Leu A and Lambert SM. Instability of the sternoclavicular joint: current concepts in classification, treatment and outcomes. Bone Joint $J$ 2013; 95: 721-731. 
27. Abiddin Z, Sinopidis C, Grocock CJ, Yin Q and Frostick SP. Suture anchors for treatment of sternoclavicular joint instability. J Shoulder Elbow Surg 2006; 15: 315-318.

28. Franck WM, Jannasch O, Siassi $M$ and Hennig FF. Balser plate stabilization: an alternate therapy for traumatic sternoclavicular instability. J Shoulder Elbow Surg 2003; 12: 276-281.

29. Martínez A, Rodríguez A, González G, Herrera A and Domingo J. Atraumatic spontaneous posterior subluxation of the sternoclavicular joint. Arch Orthop Trauma Surg 1999; 119: 344-346.

30. Bae DS, Kocher MS, Waters PM, Micheli LM, Griffey $\mathrm{M}$ and Dichtel L. Chronic recurrent anterior sternoclavicular joint instability: results of surgical management. J Pediatr Orthop 2006; 26: 71-74.

31. Blundell Bankart AS. An operation for recurrent dislocation (subluxation) of the sternoclavicular joint. Br J Surg 1938; 26: 320-323.

32. Booth CM and Roper BA. Chronic dislocation of the sternoclavicular joint: an operative repair. Clin Orthop Relat Res 1979; 140: 17-20.

33. Lyons FA1 and Rockwood CA Jr. Migration of pins used in operations on the shoulder. J Bone Joint Surg Am 1990; 72: 1262-1267.

34. Burrows HJ. Tenodesis of subclavius in the treatment of recurrent dislocation of the sterno-clavicular joint. $J$ Bone Joint Surg Br 1951; 33: 240-243.

35. Bontempo $\mathrm{N}$ a and Mazzocca AD. Biomechanics and treatment of acromioclavicular and sternoclavicular joint injuries. Br J Sports Med 2010; 44: 361-369.

36. MacDonald PB and Lapointe P. Acromioclavicular and sternoclavicular joint injuries. Orthop Clin North Am 2008; 39: 535-545.

37. Perdreau A, Bingen B, Gossing L, Lejeune É and Beugnies A. Posterior sternoclavicular epiphyseal fracture-dislocation: case report and review of literature. Inj Extra 2014; 45: 1-5.

38. Spencer EE and Kuhn JE. Biomechanical analysis of reconstructions for sternoclavicular joint instability. J Bone Joint Surg Am 2004; 86A: 98-105.

39. Kier R, Wain SL, Apple J and Martinez S. Osteoathritis of the sternoclavicular joint; radiographic features and pathological correlation. Invest Radiol 1986; 21: 227-233.

40. Lawrence CR, East B, Rashid A and Tytherleigh-Strong GM. The prevalence of osteoarthritis of the sternoclavicular joint on computed tomography. J Shoulder Elbow Surg 2017; 26: e18-e22.

41. Tytherleigh-Strong G and Griffith D. Arthroscopic excision of the sternoclavicular joint for the treatment of sternoclavicular osteoarthritis. Arthroscopy 2013; 29: 1487-1491.

42. Meis RC, Love RB, Keene JS and Orwin JF. Operative treatment of the painful sternoclavicular joint: a new technique using interpositional arthroplasty. J Shoulder Elbow Surg 2006; 15: 60-66.

43. Katthagen JC, Tahal DS, Menge TJ, Horan MP and Millett PJ. Minimum 2-year outcomes and return to sport following resection arthroplasty for the treatment of sternoclavicular osteoarthritis. J Shoulder Elbow Surg 2017; 26: e37-e43.
44. Noble JS. Degenerative sternoclavicular arthritis and hyperostosis. Clin Sports Med 2003; 22: 407-422.

45. Rockwood CA Jr, Groh GI, Wirth MA and Grassi FA. Resection arthroplasty of the sternoclavicular joint. J Bone Joint Surg Am 1997; 79A: 387-393.

46. Spar I. Psoriatic arthritis of the sternoclavicular joint. Conn Med 1978; 42: 225-226.

47. Reuler JB, Girard DE and Nardone DA. Sternoclavicular joint involvement in ankylosing spondylitis. South Med J 1978; 71: 1480-1481.

48. Taccari E, Spadaro A, Riccieri V, et al. Sternoclavicular joint disease in psoriatic arthritis. Ann Rheum Dis 1992; 51: 372-374.

49. Emery RJ, Ho EK and Leong JC. The shoulder girdle in ankylosing spondylitis. J Bone Joint Surg Am 1991; 73A: 1526-1531.

50. Richmann KM, Boutin RD, Vaughan LM, Haghighi $P$ and Resnick D. Tophaceous pseudogout of the sternoclavicular joint. AJR Am J Roentgenol 1999; 172: 1587-1589.

51. Chigira $\mathrm{M}$ and Shimizu T. Computed tomographic appearances of sternocostoclavicular hyperostosis. Skeletal Radiol 1989; 18: 347-352.

52. Freyschmidt $\mathrm{J}$ and Sternberg A. The bullhead sign: scintigraphic pattern of sternocostoclavicular hyperostosis and pustulotic arthroosteitis. Eur Radiol 1998; 8: 807-812.

53. Arlet $\mathrm{J}$ and Ficat P. Osteo-arthritis of the sternoclavicular joint. Ann Rheum Dis 1958; 17: 97-100.

54. Shen K, Yang C-L, Yin G and Xie Q-B. Sacroiliitis and spondylitis with sternoclavicular hyperostosis: SAPHO or an ankylosing spondylitis variant? Chin Med J (Engl) 2016; 129: 110-111.

55. Hayem G, Bouchaud-Chabot A, Benali K, et al. SAPHO syndrome: a long-term follow-up study of 120 cases. Semin Arthritis Rheum 1999; 29: 159-171.

56. Hukuda S, Minami M, Saito $\mathrm{T}$, et al. Spondyloarthropathies in Japan: Nationwide questionnaire survey performed by the Japan ankylosing spondylitis society. J Rheumatol 2001; 28: 554-559.

57. Takigawa T, Tanaka M, Nakanishi K, et al. SAPHO syndrome associated spondylitis. Eur Spine J 2008; 17 : 1391-1397.

58. Maugars Y, Berthelot JM, Ducloux JM and Prost A. SAPHO syndrome: a followup study of 19 cases with special emphasis on enthesis involvement. J Rheumatol 1995; 22: 2135-2141.

59. Henriques CC, Sousa M, Panarra A and Riso N. The dark side of SAPHO syndrome. BMJ Case Rep 2011; 2011: pii: bcr1120115197.

60. Carr F. The 'hidden' SAPHO syndrome. BMJ Case Rep 2014; 2014: pii: bcr2013201665.

61. Hampton SL and Youssef H. Successful treatment of resistant SAPHO syndrome with anti-TNF therapy. BMJ Case Rep 2013; 2013: pii: bcr2012007161.

62. Greenspan A, Gerscovich E, Szabo RM and Matthews JG. Condensing osteitis of the clavicle: a rare but frequently misdiagnosed condition. AJR Am J Roentgenol 1991; 156: 1011-1015. 
63. Cone RO, Resnick D, Goergen TG, Robinson C, Vint V and Haghighi P. Condensing osteitis of the clavicle. AJR Am J Roentgenol 1983; 141: 387-388.

64. Levy M, Goldberg I, Fischel R, Frisch E and Maor P. Friedrich's disease. Aseptic necrosis of the sternal end of the clavicle. J Bone Joint Surg Br 1981; 63: 539-541.

65. Outwater E and Oates E. Condensing osteitis of the clavicle: case report and review of the literature. $\mathrm{J} \mathrm{Nucl} \mathrm{Med}$ 1988; 29: 1122-1125.

66. Sharma D, Dhiman $\mathrm{P}$, Menon $\mathrm{J}$ and Krishna KV. Sternocostoclavicular joint swelling; diagnosis of a neglected entity. Arch Bone Joint Surg 2015; 3: 94-98.

67. Kofoed $\mathrm{H}$, Thomsen $\mathrm{P}$ and Lindenberg $\mathrm{S}$. Serous synovitis of the sternoclavicular joint: differential diagnostic aspects. Scand J Rheumatol 1985; 14: 61-64.

68. Restrepo CS, Martinez S, Lemos DF, et al. Imaging appearances of the sternum and sternoclavicular joints. Radiographics 2009; 29: 839-859.

69. Joethy J, Lim CH, Koong $\mathrm{HN}$ and Tan B-K. Sternoclavicular joint infection: classification of resection defects and reconstructive algorithm. Arch Plast Surg 2012; 39: 643-648..

70. Carlos GN, Kesler KA, Coleman JJ, Broderick L, Turrentine MW and Brown JW. Aggressive surgical management of sternoclavicular joint infections. J Thorac Cardiovasc Surg 1997; 113: 242-247.

71. Song HK, Guy TS, Kaiser LR and Shrager JB. Current presentation and optimal surgical management of sternoclavicular joint infections. Ann Thorac Surg 2002; 73: 427-431.

72. Burkhart HM, Deschamps C, Allen MS, Nichols FC, Miller DL and Pairolero PC. Surgical management of sternoclavicular joint infections. $J$ Thorac Cardiovasc Surg 2003; 125: 945-949.

73. Chun JM, Kim JS, Jung HJ, et al. Resection arthroplasty for septic arthritis of the sternoclavicular joint. J Shoulder Elb Surg 2012; 21: 361-366.

74. Muesse JL, Blackmon SH, Ellsworth WA and Kim MP. Treatment of sternoclavicular joint osteomyelitis with debridement and delayed resection with muscle flap coverage improves outcomes. Surg Res Pract 2014; 2014: 2014PMC4208504. 